\title{
Many-electron transport in Aharonov-Bohm interferometers: A time-dependent density-functional study
}

\author{
A. Salman ${ }^{1,2}$, V. Kotimäki ${ }^{2}$, A. Siddiki ${ }^{3}$ and E. Räsänen ${ }^{4,2}$ \\ 1 Physics Department, Akdeniz University, Antalya, Turkey \\ 2 Nanoscience Center, Department of Physics, University of Jyväskylä, FI-40014 Jyväskylä, Finland \\ 3 Physics Department, Faculty of Sciences, Istanbul University, 34134 Vezneciler-Istanbul, Turkey \\ 4 Department of Physics, Tampere University of Technology, FI-33101 Tampere, Finland
}

Received: date / Revised version: date

\begin{abstract}
We apply time-dependent density-functional theory to study many-electron transport in Aharonov-Bohm interferometers in a non-equilibrium situation. The conductance properties in the system are complex and depend on the enclosed magnetic flux in the interferometer, the number of interacting particles, and the mutual distance of the transport channels at the points of encounter. Generally, the electron-electron interactions do not suppress the visibility of Aharonov-Bohm oscillations if the interchannel distance - determined by the positioning of the incompressible strips through the external magnetic field - is optimized. However, the interactions also impose an interesting Aharonov-Bohm phase shift with channel distances below or above the optimal one. This effect is combined with suppressed oscillation amplitudes. We analyze these effects within different approximations for the exchange-correlation potential in time-dependent density-functional theory.
\end{abstract}

PACS. XX.XX.XX No PACS code given

\section{Introduction}

Integer and fractional quantum Hall effects 1 together with the Aharonov-Bohm (AB) effect 2] enable the design of interferometry devices that can be controlled by an external magnetic field alone. Significant experimental efforts in two-dimensional (2D) systems have been made 3 , 4, 5, 6, 7,8, to exploit the AB effect in the control of electron dynamics in quantum Hall interferometers. The complexity of these systems arises from the fact that not only the quantum mechanical phase of the electrons, but their path itself, is affected by the magnetic flux. Hence, the electron transport in $\mathrm{AB}$ interferometers also provides a theoretical challenge that should be approached beyond the single-particle edge-state scheme [9] that does not take the path-dependence completely into account [10].

The characteristics of the measured AB oscillations have been analyzed with edge-channel experiments 4, 11 and simulations [12. In a recent work of some of the present authors [13] the picture was made more complete by combining full electrostatics [14] of a AB interferometer device [4] with a time-propagation of noninteracting excess electrons in the obtained incompressible strips in non-equilibrium. Clear AB oscillations were found as a function of the magnetic flux, and the visibility (amplitude) of the oscillations was found to largely depend on the width and position of the current channels.
In this work we extend a previous single-electron study [13] to the many-electron regime in the framework of timedependent density-functional theory [15, 16] (TDDFT). We consider different (albeit adiabatic) approximations for the exchange-correlation functional including the exact exchange (EXX), Hartree, and local-density approximation (LDA). We find that in the high-visibility regime, corresponding to an optimal positioning of the incompressible strips (and thus the current channels), the electronelectron interactions do not suppress the AB oscillations. This is in accordance with a previous TDDFT study on semiconductor quantum rings [17. However, the interactions introduce an $\mathrm{AB}$ phase shift whose magnitude is an increasing function of the electron number varied between one and ten. In addition, the interactions suppress the AB amplitudes if the current channels are either too close or too far apart from each other. The induced phase shift could be measured in an experiment, which would eventually quantify the actual role of electronic interactions in a real setup.

\section{Model and methods}

The transport simulation is carried out by injecting manyelectron wave packets into a static 2D potential. Figure 1 shows a schematic picture of the system. The shape of the model potential is based on the experimental AlGaAs/GaAs 


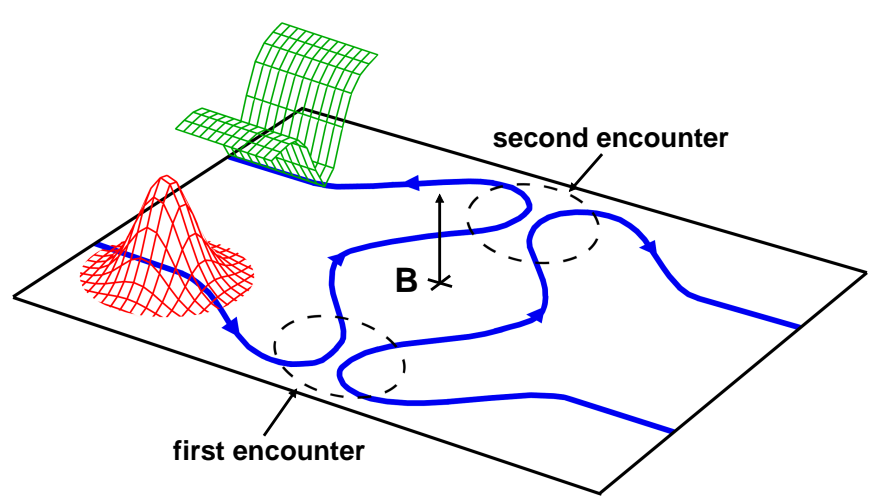

Fig. 1. (Color online) Schematic figure of the transport setup. The upper mesh represents the cross section of the model channel potential along the blue (black) thick lines. The lower mesh shows the initial wave packet used in the time-propagation.

device by Camino et al. 4 and electrostatic calculations performed in Ref. [13. In particular, the potential corresponds to incompressible strips with an integer filling factor (here $\nu=2$ ) that carry the non-equilibrium electric current. The model potential alongside the thick curves in Fig. 11 has a Gaussian cross section, $V_{\text {cross }}=-V_{0} e^{-s^{2} / c^{2}}$, where $s$ is the coordinate perpendicular to the channel, $V_{0}=80$ (Hartree atomic units used throughout) is the channel depth set to prevent the electrons from escaping the tracks, and $c=0.3$ is the track width parameter. A part of the potential is visualized as a mesh in the upper part of Fig. 1 A Gaussian cross section is a convenient choice for two reasons. First, it is a good approximation for the magnetic (parabolic) confinement at the bottom of the channel. Secondly, the upper part of the potential allows "leaking" of the electron flow in consistency with the experimental situation. It is important to note that in the time-propagation we deal with the excess electron density on top of the Fermi background that is assumed to be static.

We also apply a linear ramp potential to accelerate the initial state, thus mimicking the source-drain voltage. The initial state in the calculation is an $N$-electron wave packet at the lower-left corner of the system (see the lower mesh in Fig. 1). It is calculated by solving the groundstate problem for a part of the input channel (confined by step functions) that contains $N$ Coulomb-interacting particles [17.

Our transport scheme described above leads to inevitable non-chiral transport, i.e., the direction of the current is the same on the both sides of the interferometer. This is in accordance with non-equilibrium transport experiments [18, where an external current is imposed and a Hall (electrochemical) potential difference develops at two opposite edges with the same slope 19. Such potential drops in the incompressible strip have been found in several experiments $20,21,22$. In the conventional equilibrium case, however, the current would be chiral as confirmed in several studies.

In the time propagation of electrons we apply TDDFT [15First we consider electron transport in a two-electron sys16. on a $2 \mathrm{D}$ real-space grid. We use the fourth-order Tay- lor expansion of the time evolution operator to the KohnSham states and perform the calculation with the OCTOPUS code package 23 . The exchange-correlation potential in TDDFT is treated within adiabatic LDA, and in the two-particle case also with the (adiabatic) exact exchange approximation. In comparison, we have applied the bare Hartree approximation (including only classical electrostatic interactions and neglecting exchange and correlation) and independent-electron approximation (neglecting interactions completely).

We consider the conductance of the system as a function of the uniform and perpendicular magnetic-field strength in the units of the enclosed flux quanta, $\Phi / \Phi_{0}$, where $\Phi=B \times$ area and $\Phi_{0}=h / e$. Following the most experimental setups, the magnetic field is homogeneously distributed across the whole system. Another changing variable is the distance between the points of encounter injected particles $N=2,6$, or 10 . We consider only spincompensated (zero total spin) systems. We point out that in an experimental situation $d$ would be essentially controlled either with the gates, or with the magnetic field that affects the position and the width of the incompressible strips.

We compute the area of the interferometer by integrating the region enclosed by the curve drawn on the plane by the minima of $V_{\text {cross }}$, and cut at the points of encounter (see Fig. 11). Therefore, varying interchannel distance $d$ is taken into account in the calculation of the flux. However, the magnetic field affects only the flux but not the shape of the channels. This is justified by the following consideration. In a typical situation, notable changes in the spatial distribution of the incompressible strips can be seen in the scale of $\Delta B \sim 0.1 \ldots 0.4 \mathrm{~T}$ (see Fig. 2 in Ref. [13). However, with the dimensions of a typical sample in a micrometer range, an increase (decrease) of the field strength by this amount would add (remove) about $100 \ldots 400$ flux quanta. Therefore, as we consider changes within only three flux quanta, the shape of the channels can be kept fixed by a good approximation.

The relative conductance is estimated as the number of electrons found in the top-right corner of the system as a function of time [17. Since the simulation box is finite, we monitor the electron density only until back-scattering effects from the borders of the box appear. The measured conductance parameter is the normalized probability density found in the upper right corner of the system. We point out that the setup is not symmetric with respect to the electron current; an opposite direction of the magnetic field $(\mathbf{B} \rightarrow-\mathbf{B})$ would yield different results. Thus the situation is different from a typical symmetric transport experiment with a single input and a single output lead. A more detailed discussion of the model system can be found in Ref. [13].

\section{Results} tem. The initial state is injected in the interferometer us(interchannel distance) $d$ (see Fig. 1) and the number of 

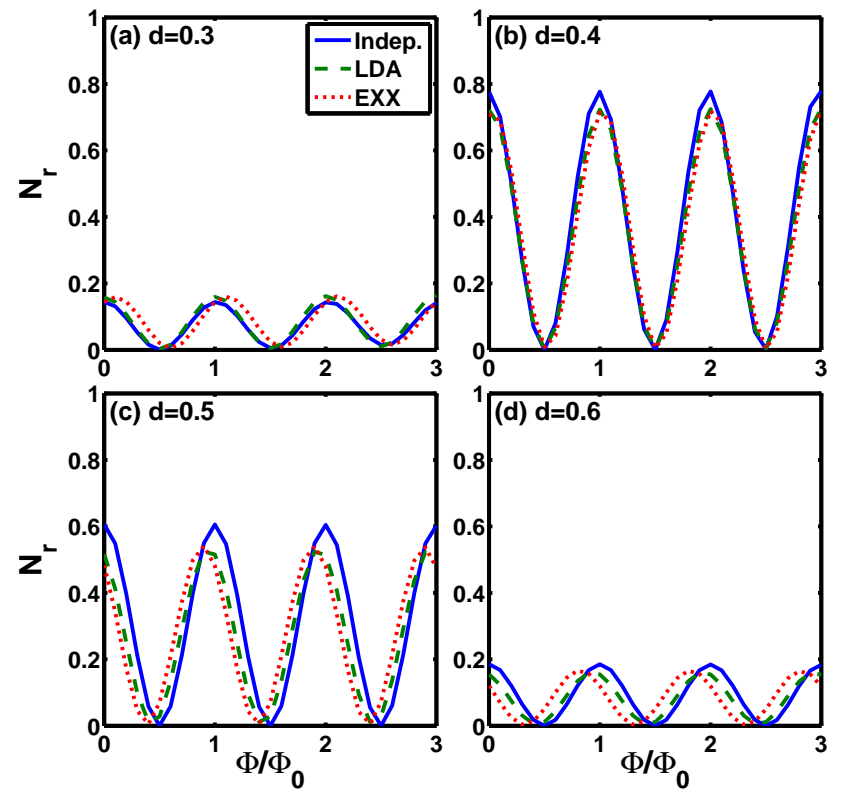

Fig. 2. (Color online) Estimated conductance in two-electron Aharonov-Bohm interferometers with different interchannel distances and different approximations within time-dependent density-functional theory (independent electrons, adiabatic local-density approximation, adiabatic exact-exchange calculation). The maximum visibility is obtained with $d \sim 0.4$.

ing the linear ramp potential, and the relative conductance is assessed as described in the previous section. The panels in Fig. 2 show the conductance for different interchannel distances, respectively, and different approximations for the electron-electron interactions are compared.

In all cases we find distinctive $\mathrm{AB}$ oscillations with the correct flux quantization in the noninteracting limit. Hence, the direction of the electron current (to the left or right drain) is completely controlled by the flux. The maximum amplitude (visibility) of the AB oscillations strongly depends on the interchannel distance. Regardless of the interactions, the maximum visibility is found at $d \sim 0.4$. A detailed look in the electron densities show that in this case the partitioning of the wave packet in the first point of encounter (see Fig. 1) is close to even, i.e., one electron remains on the left track, but the other one is transported to the right track. Consequently, the interference at the second point of encounter is as complete as possible.

For two electrons the interactions seem to have a minor effect on transport characteristics. They only induce a small phase shift in the $\mathrm{AB}$ oscillations. The direction of the phase shift depends on $d$ : at $d \lesssim 0.4$ (below the maximum visibility) the oscillations are shifted to higher $\Phi / \Phi_{0}$ due to interactions, and at $d \gtrsim 0.4$ the situation is opposite. The phase shifts are analyzed in more detail below within Fig. 6.

Moreover, as seen in Fig. 2, the EXX and LDA results are generally very close to each other. As the electronic correlations are expected to be relatively small, the obtained agreement validates the accuracy of LDA (as in EXX the exchange part is exact). In this respect, our dy-
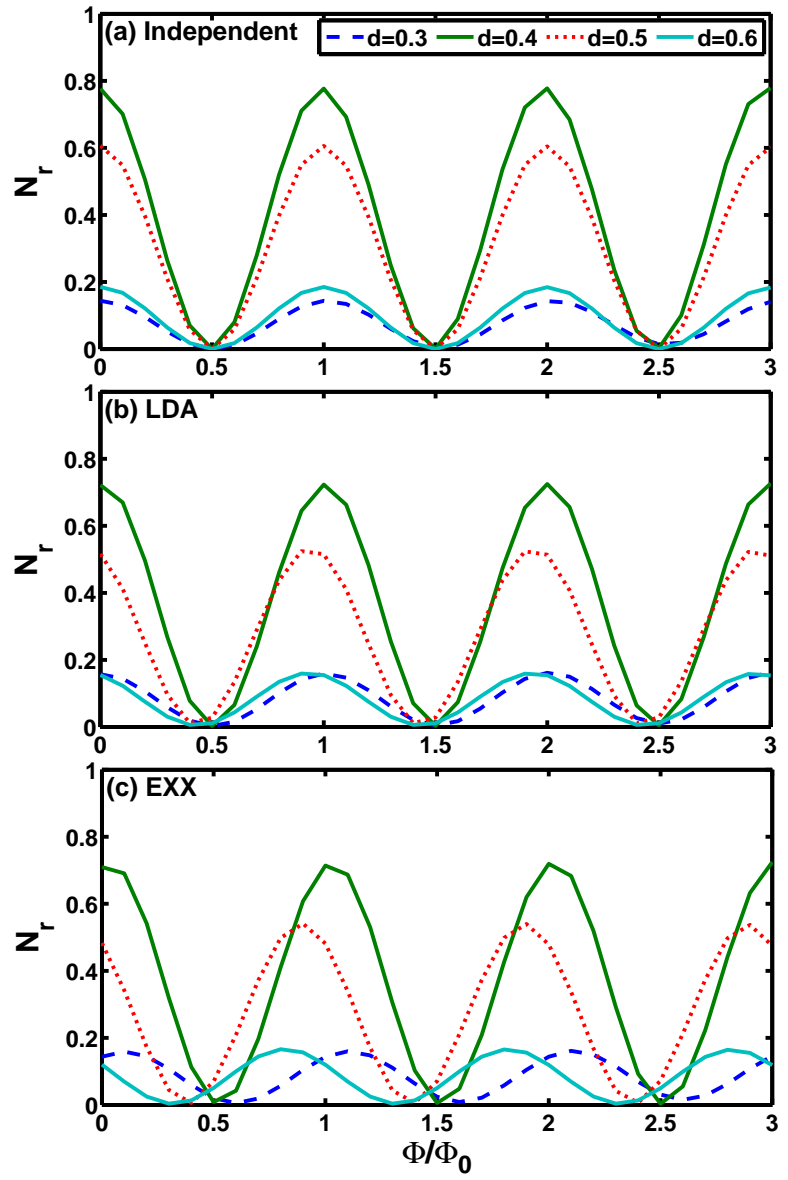

Fig. 3. (Color online) Same two-electron data as in Fig. 2 but plotted separately for different approximations within timedependent density-functional theory.

namical results are in accordance with previous densityfunctional studies where the (surprisingly) high accuracy of LDA results have been pointed out - even in complicated situations such as quantum Hall droplets with fractional filling factors such as $\nu=5 / 2$ (Refs. [24,25, 26]) and $\nu=1 / 3$ (Ref. [27]).

In Fig. 3 we plot the same data as in Fig. 2 but separate the results in terms of the interaction scheme. The homogeneity of the $\mathrm{AB}$ oscillations in the noninteracting case (a) is very clear, so that only the amplitude changes as a function of $d$. As discussed above, LDA and EXX results are similar and both show considerable phase shifts as a function of $d$.

Next we consider the physical mechanism behind the phase shift induced by interactions in Fig. 3. The Coulomb repulsion enhances the spreading of the wave packet. This occurs in an asymmetric fashion, so that there is relatively more spreading on the side of the interferometer where the electron density is higher. As the spreading is also radial, the outer parts of the wave packet capture a larger phase than the inner parts. This effect eventually leads to a shift in the $\mathrm{AB}$ oscillation phase when the interactions are present. 
In Fig. 4 we plot the conductance as a function of the flux for a ten-electron system. The results with distinctive $\mathrm{AB}$ oscillations are qualitatively similar to the two-electron situation considered above. However, there are some important differences. First, the maximum amplitude is obtained with a similar interchannel distance $(d \sim 0.4)$ as in the two-electron case. This is due to the maximum partitioning (50\% vs. $50 \%$ ) to the left and right channels with this $d$, so that the underlying mechanism is similar to the two-electron system. Secondly, we find that apart from the $d=0.4$ case the amplitude is significantly dampened by the electron-electron interactions. Thirdly, as shown in Fig. 5, also the AB phase is affected by the interactions more than in the two-electron system. This can be understood by the fact that the density cloud is larger, so that its parts capture different phases. Consequently, the overall amplitude is partly smeared out. It is noteworthy that in the case of $d=0.6$ the $\mathrm{AB}$ oscillations are almost completely dampened in the LDA solution [see Fig. [5(b)].

EXX calculations are not numerically stable for tenelectron systems considered in Figs. 4 and 5, However, we compare the adiabatic LDA calculations with the Hartree results, where the exchange-correlation effects are omitted. The results in the high-amplitude regime are similar, and also generally the deviations are relatively modest. Overall, the high-amplitude (high-visibility) regime is not considerably affected by the electron-electron interactions. Therefore, we might expect that in a real experimental setup the $\mathrm{AB}$ oscillations are always present if the partitioning is optimal, which is eventually determined by the location of the incompressible strips controlled by the initial magnetic field. Moreover, the AB phase can be controlled by changing the positioning of the incompressible strips; however, the phase change is achieved only with the price of suppressing the visibility.

In Fig. 6 we present a summarized visualization of the $\mathrm{AB}$ oscillation visibility with different number of electrons propagating in the interferometer, (a) $N=1$, (b) $N=6$, and (c) $N=10$. For the six- and ten-electron results we use the adiabatic LDA. Our results demonstrate that, as discussed above, the overall visibility is not reduced as a function of $N$. Moreover, unless the interchannel distance is close to the optimal value $d \sim 0.4$, the interactions reduce the $\mathrm{AB}$ oscillation amplitudes. This is visualized in Fig. 7 where we plot the maximum amplitude in the whole range $\Phi / \Phi_{0}=0 \ldots 3$. We find that in the interacting $N=10$ case the amplitude decays close to zero at $d=0.6$, whereas at that distance the single-electron result - and the $N=10$ without electron-electron interactions - still have a $20 \%$ visibility.

\section{Summary and discussion}

In summary, we have applied a single-electron propagation scheme as well as time-dependent density functional theory to study electron transport in realistic AharanovBohm interferometers in the integer filling factor regime.
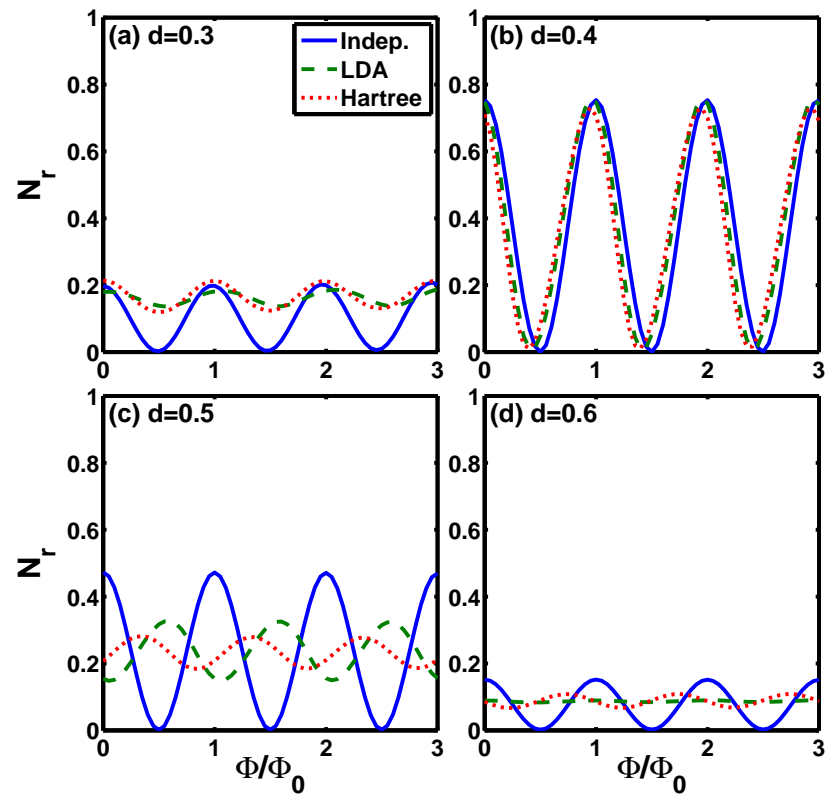

Fig. 4. (Color online) Estimated conductance in ten-electron Aharonov-Bohm interferometers with different interchannel distances and different approximations within time-dependent density-functional theory (independent electrons, adiabatic local-density approximation, Hartree approximation).

The device geometry has been obtained from experimental boundary conditions that have been applied in a selfconsistent Poisson solver within the Thomas-Fermi approximation in order to obtain the positions of the incompressible strips. Here we have assumed, in accordance with previous experiments and calculations, that the electric current is carried along the incompressible strips in a non-chiral fashion (as the system is in non-equilibrium). The current channels have been modeled with a Gaussian tubes where electronic wave packets have been injected.

The time-evolution of excess electron density leads to distinctive Aharonov-Bohm oscillations as a function of magnetic flux. The nature of the oscillations depend on the electron-electron interactions handled within (adiabatic) exact-exchange, local-density approximation, and Hartree approximation, respectively, in time-dependent densityfunctional theory. We have found that the local-density approximation is accurate in comparison with the exactexchange approach.

The electron-electron interactions do not affect the maximum visibility even if the number of propagating electrons is increased to ten. This is in agreement with a previous time-dependent density-functional study on manyelectron semiconductor quantum rings [17. However, we have found that around the optimal interchannel distance the interactions induce a phase shift in the AharonovBohm oscillations, and the strength of the shift is proportional to the number of electrons. If the interchannel distance is far from optimal the interactions may dampen the Aharonov-Bohm oscillations completely.

Our results confirm that in a corresponding experimental setup the Aharonov-Bohm oscillations should be 

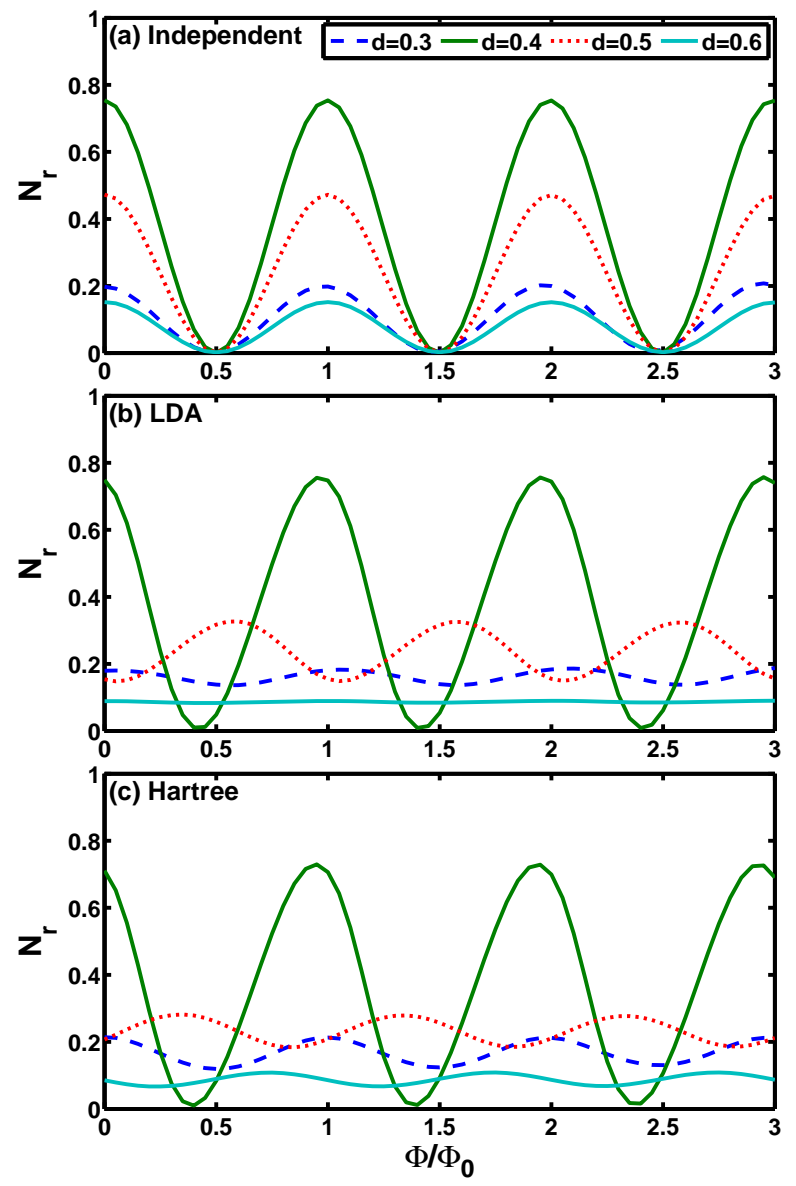

Fig. 5. (Color online) Same ten-electron data as in Fig. 4 but now plotted separately for different approximations within time-dependent density-functional theory.

clearly visible, especially if the incompressible strips are sufficiently narrow. Furthermore, possible phase shifts in the oscillations in different magnetic-field ranges, i.e., with different positions and widths of the incompressible strips, could reveal the importance of interaction effects in a real setup. As a signature of such a dependency on the interchannel distance we would like to mention the early experiments performed in Mach-Zehnder geometry [11. There the visibility of Aharonov-Bohm oscillations is suppressed depending on the current excitation, and this phenomena is attributed to the energy dependence of the transmission probabilities. We hope that the present study encourages further experimental efforts in this direction.

This work was supported by TÜBİTAK under grant no. 109T083, Istanbul University (BAP:6970), Magnus Ehrnrooth Foundation, ERASMUS Exchange Programme, Acadeng of Finland, and Institute of Theoretical and Applied Physics (ITAP) in Turunç, Turkey. CSC Scientific Computing Ltd. is acknowledged for computational resources.
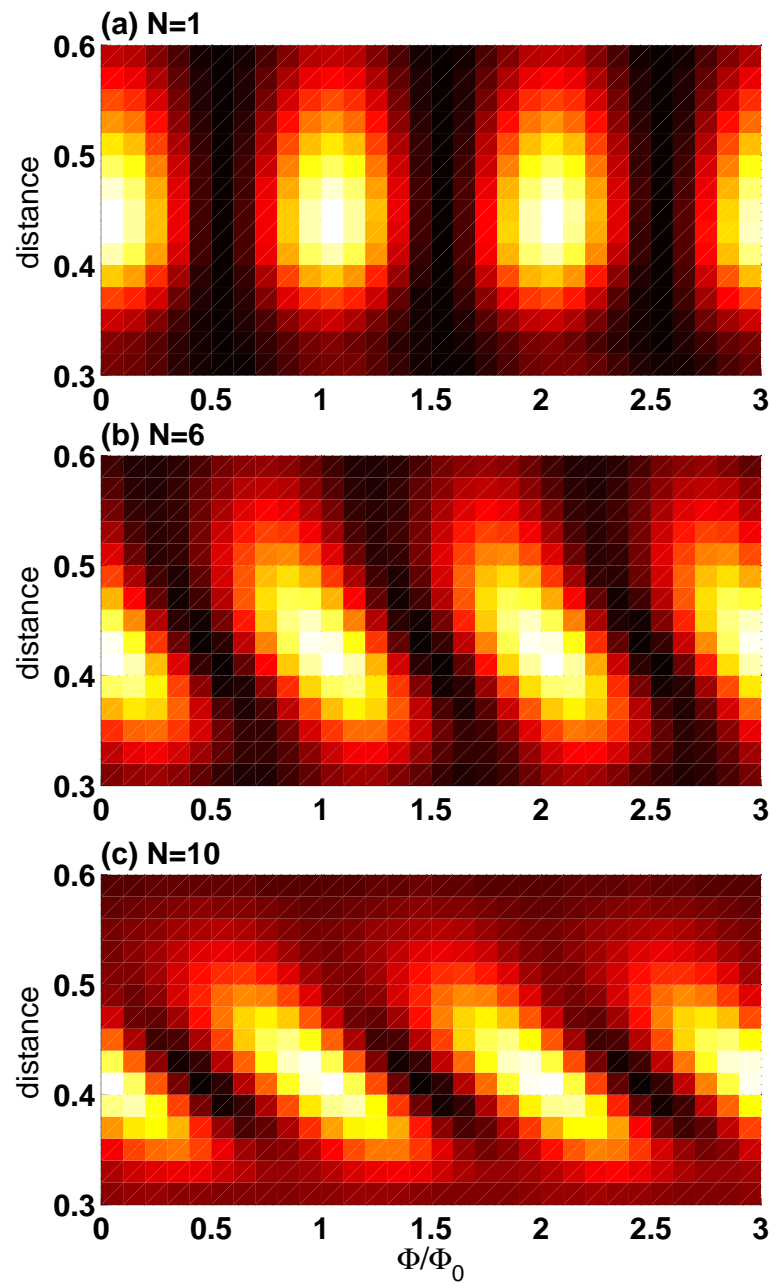

Fig. 6. (Color online) Aharonov-Bohm oscillations (color scale) for one-, six-, and ten-electron systems, respectively. Clear phase shifts can be in many-electron systems treated here within adiabatic local-density approximation.

\section{References}

1. T. Chakraborty and P. Pietiläinen, The Quantum Hall Effects: Fractional and Integral (Springer, Berlin, 1995).

2. Y. Aharonov and D. Bohm, Phys. Rev. 115, 485 (1959).

3. M. Avinun-Kalish, M. Heiblum, O. Zarchin, D. Mahalu, and V. Umansky, Nature 436, 529 (2005).

4. F. E. Camino, W. Zhou, and V. J. Goldman, Phys. Rev B 72, 155313 (2005).

5. N. Ofek, A. Bid, M. Heiblum, A. Stern, V. Umansky, and D. Mahalu, Proceedings of the National Academy of Science 107, 5276 (2010).

6. M. D. Godfrey, P. Jiang, W. Kang, S. H. Simon, K. W. Baldwin, L. N. Pfeiffer, and K. W. West, ArXiv e-prints (2007), 0708.2448.

7. D. T. McClure, Y. Zhang, B. Rosenow, E. M. LevensonFalk, C. M. Marcus, L. N. Pfeiffer, and K. W. West, Phys. Rev. Lett. 103, 206806 (2009).

y. Zhang, D. T. McClure, E. M. Levenson-Falk, C. M. Marcus, L. N. Pfeiffer, and K. W. West, Phys. Rev. B 79, 241304 (2009).

9. M. Büttiker, Phys. Rev. Lett. 57, 1761 (1986). 


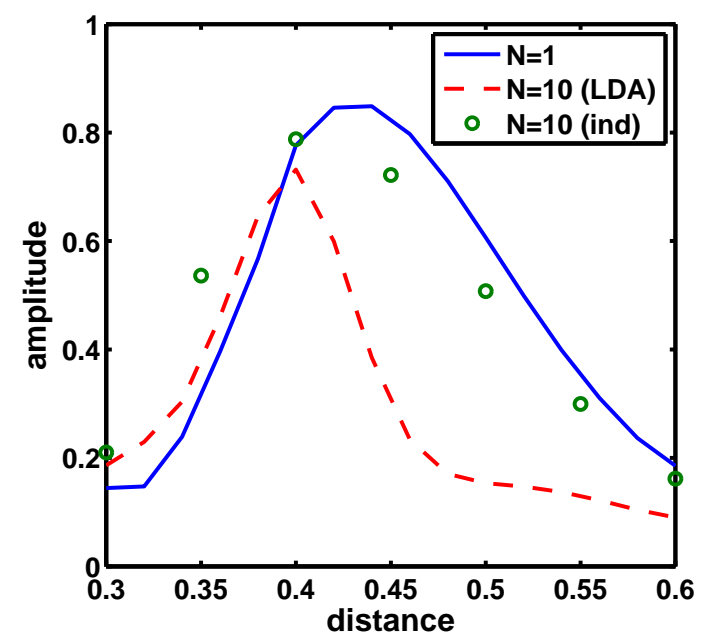

Fig. 7. (Color online) Maximum amplitude of the AharonovBohm oscillations (from minimum to maximum conductance) in the range $\Phi / \Phi_{0}=0 \ldots 3$ in the case of $N=1$ (solid line), $N=10$ caluculated with the adiabatic local-density approximation (dashed line) and without interactions (circles).

10. B. Rosenow and B. I. Halperin, Phys. Rev. Lett. 98, 106801 (2007)

11. I. Neder, M. Heiblum, Y. Levinson, D. Mahalu, and V. Umansky, Phys. Rev. Lett. 96, 016804 (2006).

12. S. Ihnatsenka and I. V. Zozoulenko, Phys. Rev. B 77, 235304 (2008).

13. V. Kotimäki, E. Cicek, A. Siddiki, and E. Räsänen, New J. Phys. 14, 053024 (2012).

14. A. Siddiki and K. Guven, arXiv:1006.5012 (2010).

15. C. Ullrich, Time-Dependent Density-Functional Theory: Concepts and Applications, (Oxford University Press, 2012).

16. Time-Dependent Density Functional Theory, Lecture Notes in Physics, edited by M. A. L. Marques, C. A. Ullrich, F. Nogueira, A. Rubio, K. Burke, and E. K. U. Gross (Springer, Berlin, 2006).

17. V. Kotimäki and E. Räsänen, Phys. Rev. B 81, 245316 (2010).

18. A. Siddiki, J. Horas, D. Kupidura, W. Wegscheider, and S. Ludwig, New J. Phys. 12, 113011 (2010).

19. K. Güven and R. R. Gerhardts, Phys. Rev. B 67, 115327 (2003).

20. E. Ahlswede, P. Weitz, J. Weis, K. von Klitzing, and K. Eberl, Physica B 298, 562 (2001).

21. E. Ahlswede, J. Weis, K. von Klitzing, and K. Eberl, Physica E 12, 165 (2002).

22. F. Dahlem, E. Ahlswede, J. Weis, and K. von Klitzing, Phys. Rev. B 82, 121305 (2010).

23. M. A. L. Marques, A. Castro, G.F. Bertsch, A. Rubio, Comput. Phys. Commun. 151, 60 (2003); A. Castro, H. Appel, M. Oliveira, C. A. Rozzi, X. Andrade, F. Lorenzen, M. A. L. Marques, E. K. U. Gross, and A. Rubio, Phys. Stat. Sol. (b) 243, 2465 (2006).

24. A. Harju, H. Saarikoski, and E. Räsänen, Phys. Rev. Lett. 96, 126805 (2006).

25. E. Räsänen, H. Saarikoski, A. Harju, M. Ciorga, and A. S. Sachrajda, Phys. Rev. B 77, 041302(R) (2008).

26. M. C. Rogge, E. Räsänen, and R. J. Haug, Phys. Rev. Lett. 105, 046802 (2010).
27. H. Saarikoski, A. Harju, M. J. Puska, and R. M. Nieminen, Phys. Rev. Lett. 93, 116802 (2004). 